\title{
Neisseria Gonorrheae: A Cause of Male Infertility
}

\author{
Kumurya $\mathrm{AS}^{1}$, Bitet $\mathrm{DE}^{2 *}$ and Sani $\mathrm{A}^{1}$ \\ ${ }^{1}$ Department of Medical Laboratory Sciences, Faculty of Allied Health Sciences, College of \\ Health Science, Bayero University, Nigeria \\ ${ }^{2}$ General Hospital Kagarko, Ministry of Health and Human Services, Nigeria
}

*Corresponding author: Ezekiel Dogo Betet, General Hospital Kagarko, Ministry of Health and Human Services, Kaduna, Nigeria, Tel: 07032153561; Email: ezekieldogobetet@ymail. com

\section{Abstract}

Background: Gonorrhoea is a sexually transmitted infection that is commonly related to male infertility. The infection affects sperm transport through the urinary tract and subsequent damage of the testicular tubes. The infection also impair sperm production as the infection is rarely asymptomatic and can be difficult to diagnose, it is possible that its contribution to male infertility is underestimated. Infection of the genitals results in a purulent (pus-like) discharge from the genitals which may be foul smelling, inflammation, redness, swelling, dysuria, and a burning sensation during urination. As with Chlamydia, it is possible to have a Gonorrhea infection without noticeable symptoms, and which can cause permanent scarring and blockage in the sperm production duct. Gonorrhoea is a bacterial infection and is treatable with antibiotics. Laboratory studies reveal that N. gonorrhoeae infection can impair motility, viability and spermatogenesis; increase anti-sperm antibodies are associated with a decrease in semen parameters as a result of the production of anti-sperm antibodies in the genital tract.

Aim: The study aimed at reviewing the possible role of gonorrhoea in male infertility.

Method: Research publications such as Pubmed, Scopus, Medline etc.

Results: over 115 journals of international repute were obtained, out of which 50 were found to be closely relevant such as; the implication of $N$. gonorrheae in male infertility, complication of $N$. gonorrhea infection, $N$. gonorrheae and male infertility and were reviewed.

Conclusion: N. gonorrhoae was found implicative in male infertility and the need for comprehensive modern laboratory methods for the diagnosis of the infection and also to included routine laboratory tests.

Keywords: N. Gonorrhea; Male Infertility; Urethritis; Epididymitis; Epididymal-Orchitis

Abbreviations: WW1: World War One; HIV: Human Immunodeficiency Virus; STI: Sexually Transmitted Infection; PMN: Polymorph Nuclear Leukocyte; TNF: Tumour Necrosis Factor; LPS: Lipopolysaccharide; PID: Pelvic Inflammatory Disease; OA: Obstructive Azoospermia; IF: Immunofluorescent; CF: Complement Fixation; DFA: Direct Fluorescent Antibody Stain; EIA: Enzyme Immunoassays; Naats: Nucleic Acid Amplification Tests; PCR: Polymerase Chain Reaction.

\section{Introduction}

\section{Background History}

Antediluvians Egyptian, Chinese, Greek along with Roman literature as well as the Bible, all described symptoms related to gonorrhoea [1] and the term gonorrhoea is rooted from ancient Greek and was first used by Galen in 130 A.D, meaning the flow of seed. Albert Neisser was the first 


\section{Open Access Journal of Microbiology \& Biotechnology}

to identify the aetiological agent of gonorrhoea in 1879 in microscopy of stained smears from vaginal, urethral and conjunctival exudates [2] and termed it Micrococcus gonorrhoeae. Many other terms have historically been used for Neisseria gonorrhoeae; $[3,4]$. Previous to the discovery of operational antimicrobial therapy of $\mathrm{N}$. gonorrhoea, urethral irrigation, asceticism from alcohol and sexual activity, rest and systemic treatment with a range of balsams were the conventional therapy regimens [5]. Throughout World War One (WW1), prophylactic packets were given to soldiers, containing condoms, calomel cream and Argyrol [6]. After the successful culturing of N. gonorrhoeae by Leistikow in 1882 duo with the discovery of Gram's staining by Gram in 1884, and carbohydrate oxidation test by Elser and Huntoon in 1909, diagnosis of gonorrhoea improved significantly. The discovery of sulphonamides for the management of gonorrhoea soon after penicillin, the prospect of eradicating gonorrhoea was high, resulting in the rapid decline of gonorrhoea occurrence [6-8]. It was soon discovered that $N$. gonorrhoea has a remarkable capability to adapt to and outlive any antimicrobial therapy, and continues as a key sexually transmitted infection (STI) [9]. In the early 1960s, this trend reversed with the inception of oral contraceptive methods, and N. gonorrhoea infections reached a climax incidence of over one million reported cases in 1978 in the United States [9]. In the late 1980s, with the outbreak of the Human Immunodeficiency Virus (HIV) and concurrent prevalent use of barrier contraceptives, the incidence of gonococcal infection again declined [10]. The contemporary upsurge in all sexually transmitted diseases is presently observed globally, as a result of the emergence of antibiotic resistance amid organisms causing these diseases [11].

Sexual contact vaginally, anally or orally with an infected person serves as the basic mode of $N$. gonorrhoeae transmission; conversely, pregnant women with the gonococcal disease can transmit this bacterium to their offspring during birth $[12,13]$. Successful colonization by the gonococcus results in a broad spectrum of clinical manifestations of the disease that are, in part, determined by the particular strain that initiates the diseased state and the anatomical site of infection.

Currently, N. gonorrhoeae is highly sensitive to zoliflodacin and gepotidacin which are newly developed antibiotics. Nonetheless, the gonococcus easily develops resistance to antimicrobials, so how long these antimicrobials will remain potent is only time can reveal [14]. There is an alarming increase resistant of $N$. gonorrhoea to many antibiotics and conversely increase in male infertility globally [15]. Therefore, the goal of this appraisal is to present contemporary information on Neisserial infection as one of the causes of male infertility. Pub Med, Medline and Science Direct databases were used as the search engine for published articles related to $N$. gonorrhoeae as a cause of male fertility.

\section{Gonococcal Pathogenesis}

$N$. gonorrhoeae is closely related to $N$. meningitides and typically colonizes the mucosal epithelia of the male urethra and the female uterine cervix, but infections can also occur on the rectum, the throat and the conjunctiva of the eye. The latter can easily happen to newborns if the mother carries the pathogen, $N$. gonorrhoeae transmission generally occurs through direct vaginal, anal and oral sexual contact, but indirect modes of transmission have been reported leading to establishment of urogenital tracts infection through interaction with non-ciliated epithelial cells resulting in cellular inversion [16]. Attachment to the mucosal epithelium is followed within 24-48 hours by penetration of the organism between and through epithelial cells to the sub mucosal tissues. This is followed by massive infiltration of the infected epithelial tissue by neutrophils, resulting in the development of sub mucosal micro abscesses and exudation of pus [17].

Although different molecular mechanisms are involved during the establishment of gonococci on the mucosal surfaces of males and females, infection often leads to inflammation and polymorph nuclear leukocyte (PMN) influx. Both tumour necrosis factor (TNF) from phagocytes and gonococcal products, such as peptidoglycan and lipopolysaccharide (LPS), also cause toxic damage to ciliated epithelial cells of mucosal surfaces [18].

\section{Epidemiology}

Averagely, 62 million new cases of gonorrhoea are been reported annually worldwide with the greatest number of cases occurring in South and Southeast Asia and SubSaharan Africa [19]. However, the occurrence of new cases is still prevalent among construction workers globally and in heavily industrialized regions including Eastern and Central Europe, North America, and Western Europe [19]. Gonorrhea rates declined globally in the 1940s and 1950s with the advent of penicillin therapy [20], but began to rise again between 1966 and 1975 as a result of the changing sexual behavior patterns among people and increasing resistance to penicillin $G[19,21]$. In response to this, public health officials and health care providers launched a national campaign to control the spread of gonorrhoea through the widespread screening of asymptomatic women using newly developed selective culture media (Thayer-Martin). This approach and the increasing use of condoms were largely successful, yielding a decline in the number of diagnosed gonorrhoea cases [19,21]. Nonetheless, the number of cases of gonorrhoea increases again between 2005 and 


\section{Open Access Journal of Microbiology \& Biotechnology}

2007 globally, with the overall current rate of gonorrhoea transmission remains high.

West Africa has recorded a low prevalence of both Chlamydia trachomatis and $N$. gonorrhoeae when compared with East Africa and the Western world [22,23]. Women presenting with vaginal discharge in six countries in West Africa (Benin, Burkina Faso, Ghana, Guinea, Uganda, and Mali), tested a $3.2 \%$ prevalence [24]. A recent study conducted by Yirenya-Tawiah and others to investigate the prevalence of $C$. trachomatis and $N$. gonorrhoeae in women of reproductive age living in urogenital schistosomiasis endemic areas in Ghana showed $6.3 \%$ and $2.6 \%$ positivity of Chlamydia and Gonorrhea respectively. Samples were examined using PCR incorporating the use of Cobas Amplicor from Roche Diagnostics. They concluded that women with a history of urogenital schistosomiasis were at a higher risk of contracting the diseases [25]. Many studies have shown that infections of the reproductive tract both in men and women may impair reproductive function. However, some studies also showed the Prevalence rate of N. gonorrhoeae infection amongst undergraduate female students in the University of Port Harcourt between January and June 2009 was 5\% [26]. This result is comparable to those of Franceschi and Gopal et al. who had $2.6 \%$ and $3.8 \%$ respectively.

\section{Risk Factors}

Neisseria gonorrhoeae (gonococcus) is the etiologic agent of gonorrhea [27]. Gonorrhoea, the infection caused by the gonococcus, is the second most commonly reported notifiable disease in the United States today (CDC, 2007). Studies indicate that the mechanisms of infection differ in men and women $[7,28]$. Therefore, younger age, new sex partner, sex partner who has concurrent partners, multiple sex partners, previous history of gonorrhea infection and having other sexually transmitted infections are some of the most common risk factors of contracting gonorrhoea infection, others include immune compromised individual, prostitution, illicit drug abuse, low socioeconomic status, early onset of sexual activity, unmarried status and genetic factors [29-32].

\section{Impact on Male Reproduction}

The term gonorrhoea, meaning "flow of semen," was first termed by Galen in $130 \mathrm{AD}$, who presumed that the urethral exudate of infected males was semen [27]. The impact on fertility has been well established. In fact, in women, $N$. gonorrhoea infection may cause pelvic inflammatory disease (PID) and lead to chronic pelvic pain, ectopic pregnancy, and infertility. CDC, 2009 [33]. N. gonorrhoeae is also known to be involved in damage to the female reproductive tract and has been recognized as a cause of PID, together with
Gardnerella vaginalis and Trichomonas vaginalis [34,35]. Some studies showed that N. gonorrhoea, other bacteria, yeasts, and protozoa may interact directly with sperm. These interactions result in attachment between bacteria and sperm, agglutination phenomena and morphological alterations to sperm [36]. Furthermore, the presence of pathogenic bacteria in the vagina has been associated with the release of pro-inflammatory cytokines, and a correlation between elevated IL-beta and IL-8 and idiopathic fertility was demonstrated [34].

Men who become infected with $N$. gonorrhoeae usually present with acute anterior urethritis, prostatitis, epididymitis, proctitis, reactive arthritis, and decreased fertility which is accompanied by symptoms of urethral discharge and dysuria $[27,7]$. Urethritis is the inflammation of the urethra (urine tube) that runs along the underside of the penis. Symptoms include a white cloudy discharge from the tip of the penis, pain or a burning sensation when the patient urinates; urge to urinate often, irritation and soreness around the tip of the penis. Although there are many causes of urethritis $N$. gonorrhoeae infection is the most common [37]. The incubation period in males is between 2-8 days, but can equally take up to 10 days or even longer $[7,15,27,38]$. However, approximately 1 per cent of men can be asymptomatic for up to 6 weeks after exposure [27]. This has been linked to infection by particular N. gonorrhoea strains, namely arginine, hypoxanthine, and uracil auxotrophs referred to as AHU- strains [39].

Prostatitis is one of the most common urological disorders and can affect men of any age. Approximately one-third of all men during their lifetime will experience symptoms consistent with prostatitis [37]. The early classification of prostatitis described four syndromes for which pelvic pain in the male was the common factor. The NIH classification system designates categories I and II for cases in which bacteria cause acute or chronic prostatitis, respectively. In acute bacterial prostatitis, patients can present with voiding complaints, such as dysuria, frequency, urgency, or hesitancy. Other symptoms can include suprapubic pain, hematuria, or systemic symptoms such as fever, chills, nausea, vomiting, or malaise $[37,40]$.

Epididymitis is a condition that causes swelling and tenderness in the epididymis. The epididymis is part of a man's reproductive system that carries sperms from the testicles, so if the testicles are affected, it is called epidymoorchitis. Gonorrhoea may cause painful testicles or infertility by blockage of epidermis or tubes, then the spermatozoa are unable to move out of the genitourinary tract causing a condition known as obstructive azoospermia (OA) which by implication means there are no spermatozoa in the ejaculated semen leading to sterility of the semen fluid [41]. 


\section{Open Access Journal of Microbiology \& Biotechnology}

Gonorrhoea infections that go undetected and untreated in men for a long time, lead to epididymitis - affecting the ducts that are attached to the testicles where spermatozoa mature. Epididymitis can cause the infected testicle to shrink, abscesses, and sores in the surrounding scrotum area, the structures of the male reproductive tract, including the epididymis and urethra, can equally be damaged by infection with untreated gonorrhoea. Although uncommon among men but has a serious impact on male infertility. Therefore, Gonorrhea complication in men can optimally cause infertility [42].

\section{Laboratory Diagnosis}

Laboratory diagnosis of N. gonorrhoeae has traditionally consisted of direct microscopy of Gram- or methylene blue stained smears of urethral specimens from men or women and culture of fastidious $N$. gonorrhoeae prepared from urogenital specimens and currently, the antigen and nucleic acid detection technologies are developed $[40,43,44]$. Antibodies to Neisseria spp. are detected with immunofluorescent (IF) assay, but these assays are expensive, low sensitivity and not widely available. The IF and complement fixation (CF) tests are not too sensitive in the diagnosis of urogenital infection. The value of Neisseriaspecific antibodies (IgM, IgG, IgA) in the diagnosis of urogenital infections using indirect immunofluorescence (IF)method is limited since these antibodies are not genusspecific and thus will also be elevated in infections with none pathogenic Neisseria strains. Concerning high specificity, the bacteriological culture has been the method of choice for diagnosis. Another advantage is that these cultures maintain the viability of the microorganisms for additional studies such as genotyping or antimicrobial susceptibility tests $[21,44,45]$. One disadvantage of culture is the low sensitivity of $70-85 \%$. Furthermore, the costs, the high level of technical expertise necessary and the time required to obtain results, are significant disadvantages of this method. Rapid methods for diagnosis of an $N$. gonorrhoeae infection include a direct fluorescent antibody stain (DFA) and enzyme immunoassays (EIA) to detect antigens of the bacterium. Both types of assay have low sensitivities compared to culture and DNA amplification methods [21]. Compared to culture, the sensitivity of these assays, depending on many variables including the population examined and the culture technique used for comparison has been reported to vary from 50 to $90 \%$. Because of their high sensitivity and specificity, and their possible use for a large range of sample types, nucleic acid amplification tests (NAATs) are the tests of choice for the diagnosis of $N$. gonorrhoeae genital infections. Several commercial NAATs are available and make use of different technologies: polymerase chain reaction (PCR) and realtime PCR, strand displacement amplification, transcriptionmediated amplification, and nucleic acid sequence-based amplification [46]. The major targets for amplification-based tests are generally multiple-copy genes, e.g. those carried by the cryptic plasmid of $N$. gonorrhoeae, or gene products such as rRNAs $[15,42,47]$.

\section{Treatment for Gonorrhea}

Many antibiotics can successfully cure gonorrhoea in adolescents and adults. However, drug-resistant strains of gonorrhoea are on the increase in many parts of the world, including the United States, West Africa, and Asia, successful treatment of gonorrhoea is becoming more difficult, because many people with gonorrhoea also have Chlamydia (another STI), antibiotics for both infections are usually given together $[21,47,48]$. Zoliflodacin and gepotidacin are new antibiotics currently effective for the treatment of $N$. gonorrhoeae infection [14].

\section{Prevention of Neisseria Gonorrhoea Infection}

The best way to avoid transmission of STIs is public health education, totally abstain from sexual intercourse before marriage and those who are married to remain faithful to their partners, or to be in a long-term mutually monogamous relationship with a partner who has been tested and is known to be uninfected [47].Latex condoms, when used consistently and correctly, can reduce the risk of transmission of gonorrhoea, also the person and all of his or her sex partners must avoid sex until they have completed their treatment for gonorrhea [48-50]. All persons at high risk of gonorrhoea infection must be screened for Chlamydia, HIV, and syphilis, above all, personal hygiene.

\section{Conclusion}

Infection with $N$. gonorrhoeae accounts for the rarely most common sexually transmitted infection globally. In men, $N$. gonorrhoeae can cause urethritis, epididymitis, epididymal- orchitis and prostatitis, although asymptomatic infections are often common. Acute and chronic infection and/ or inflammation can cause partial or complete obstruction of sperm transport leading to oligozoospermia or azoospermia respectively. Subclinical gonorrhoea infection may activate an immune response to sperm. The infection of the testis and prostate is implicated in the deterioration of sperm, possibly affecting male fertility. Also, there is increasing evidence that the function of human spermatozoa can be significantly affected by direct exposure to the bacterium or by the host immune response induced by it. The role of leukocytospermia in the pathogenesis of male infertility remains unknown. The roles in which leukocytes and $N$. gonorrhoeae may result in sperm damage involve ROS generation and the induction of sperm apoptosis. On the other hand, the co-infection with N. gonorrhoeae and other microorganisms may be a cause 


\section{Open Access Journal of Microbiology \& Biotechnology}

of the impairment of sperm quality, motility, and function. Efforts should be made at achieving proper diagnosis using a more sensitive method (NAAT), and adequate treatment is given promptly to avoid resistance and complications.

\section{Recommendations}

Screening for gonorrhea infection should be made mandatory for all intending couples and intermittently for all adults with multiple sexual partners. Modern laboratory diagnostic equipment should be made available in all STI clinics across the globe especially in developing countries. Public awareness should be taken seriously just like that of HIV/AIDS and those found with the infection should be promptly treated.

\section{References}

1. Rosebury T (1971) Microbes and morals: The strange story of venereal disease, Viking Press, New York.

2. Neisser A(1879) Ueber eine der Gonorrhoe eigentümliche Micrococcusform Centralb. Med Wissenschaften 28: 497-500.

3. Kinghorn G (2010) Pharyngeal gonorrhoea: a silent cause for concern. Sex Transm Infect 86(6): 413-414.

4. Lehmann KB, Neumann R (1896) Atlas und Grundriss der Bakteriologie und Lehrbuch der speciellen bakteriologischen Diagnostik, $1^{\text {st }}$ (Edn.), München : J F Lehmann, pp: 1-448.

5. Oriel JD (1994) The British Journal of Venereal Diseases and Genitourinary Medicine: The first 70 years. Genitourin Med 70(4): 235-239.

6. Lewis DA (2010) The Gonococcus fights back: Is this time a knockout? Sex Transm Infect 86(6): 415-421.

7. Hook EW Holmes, Sparling KK, Lemon PF, Handsfield SM (1999) Gonococcal infections in the adult. Sexually transmitted diseases, $3^{\text {rd }}$ (Edn.), McGraw-Hill, New York, pp: 451-466.

8. Mahoney JF, Ferguson C, Buchholtz M, Van Slyke CJ (1943) The use of penicillin sodium in the treatment of sulfonamide-resistant gonorrhoea in men. A preliminary report. American Journal of Syphilis 27(5): 525-528.

9. Handsfield HH, Mandell GLV, Douglas RG, Bennett JE (1990) Neisseria gonorrhoeae, Principles and practice of infectious disease, $3^{\text {rd }}$ (Edn.), Churchill Livingstone, New York, pp: 1613-1631.

10. Knapp JS, Rice RJ, Murray PR, Baron EJ, Pfaller
MA (1995) Neisseria and Branhamella, Manual of clinical microbiology, $6^{\text {th }}$ (Edn.), American Society of Microbiology Press, Washington DC, pp: 324-340.

11. Ison CA, Dillon JA, Tapsall JW (1998) The epidemiology of global antibiotic resistance among Neisseria gonorrhoeae and Haemophilus ducreyi. Lancet 351(S3): 8-11.

12. Bolan G, Ehrhardt AA, Wasserheit JN, Holmes KK, Mardh PA, et al. (1999) Gender perspectives and Sexually transmitted diseases, $3^{\text {rd }}$ (Edn.), McGraw-Hill, New York, pp: 117-127.

13. Brooks GF, Donegan EA (1985) Pathogenesis and immunology of experimental gonococcal infection. Gonococcal infection, Butler and Tanner, Ltd., London, United Kingdom, pp: 51-82.

14. Scangarella-Oman NE, Hossain M, Dixon PB, Ingraham $\mathrm{K}$, Min S, et al. (2018) Microbiological analysis phase 2 randomized study in adults evaluating single oral doses of gepotidacin in the treatment of uncomplicated urogenital gonorrhoea caused by Neisseria gonorrhoeae. Antimicrob Agents Chemother 62(12): 1221-1318.

15. WHO (2015) Global Prevalence and Incidence of Selected Curable Sexually Transmitted Infections diseases: Overview and Estimates. World Health Organization, Geneva.

16. Selvan M, Agarwal A (2018) A systematic review of sperm DNA fragmentation in male factor infertility: Laboratory assessment. Arab J Urol 16(1): 65 -76.

17. Kleist E, Moi H (1993) Transmission of gonorrhoea through an inflatable doll. Genitourin Med 69 (4): 322

18. Casey SG, Shafer WM, Spitznagel JK (1986) Neisseria gonorrhoeae survive intraleukocytic oxygenindependent antimicrobial capacities of anaerobic and aerobic granulocytes in the presence of pyocin lethal for extracellular gonococci. Infect Immun 52 (2): 384-389.

19. Edwards JL, Apicella MA (2004) The molecular mechanisms used by Neisseria gonorrhoeae to initiate infection differ between men and women. Clin Microbiol Rev 17(4): 965-981.

20. Vickerman P, Peeling RW, Watts C, Mabey D (2005) Detection of gonococcal infection: Pros and cons of a rapid test. Mol Diagn 9(4): 175-179.

21. Barbee LA, Dombrowski JC (2013) Control of Neisseria Gonorrhoeae in the Era of evolving antimicrobial Resistance. Infect Dis North Am 27(4): 723-737. 


\section{Open Access Journal of Microbiology \& Biotechnology}

22. Hook EW $3^{\text {rd }}$, Kirkcaldy RD (2018) A brief history of evolving diagnosis and therapy for Gonorrhea: Lessons learned. Clin Infect Dis 67(8): 1294-1299.

23. Torrone EA, Morrison CS, Chen PL, Kwok C, Francis SC, et al. (2018) Correction: Prevalence of sexually transmitted infections and bacterial vaginosis among women in subSaharan Africa: An individual participant data metaanalysis of 18 HIV prevention studies. PLOS Med 15(2): e1002511.

24. Ramjee G, Abbai NS, Naidoo S (2015) Women and Sexually Transmitted Infections in Africa. Open Journal of Obstetrics and Gynecology 5: 385-399.

25. Rasajo E, Tumwesigye NM, Kambugu F, Tenywa T, Darj E (2006) Prevalence of sexually transmitted infections among adolescents in Kampala, Uganda, and theoretical models for improving syndromic management. J Adolesc Health 38(3): 213-221.

26. Yirenya-Tawiah D, Annang TN, Apea-Kubi KA, Lomo G, Mensah D, et al. (2014) Chlamydia Trachomatis and Neisseria Gonorrhoeae prevalence among women of reproductive age living in urogenital schistosomiasis endemic area in Ghana. BMC Res Notes 7: 349.

27. Kennedy TW, Ibianabo LO (2013) Prevalence of Neisseria Gonorrhoea among undergraduate female students of University of Port Harcourt Using Strand Displacement and Amplification(SDA) Technique. IOSR Journal of Dental and Medical Sciences 7(4): 76-79.

28. Hansfield HH, Sparling PF, Mandell GLV (2005) Neisseria gonorrhoeae. In: Principles and Practices of Infectious Diseases, Churchill Livingstone Inc, New York, NY, pp: 2514-2529.

29. Zanetti S, Usai D, Molicotti P, Deriu A, Sechi LA, et al. (2007) Presence of Chlamydia trachomatis in young women in Northern Sardinia. New Microbiol 30(1): 6364.

30. Edwards JL, Apicella MA (2005) I-domain-containing integrins serve as pilus receptors for Neisseria gonorrhoeae adherence to human epithelial cells. Cell Microbiol 7(8): 1197-1211.

31. CDC (2018) Sexually transmitted Diseases laboratory Guidelines, MMWR, Center for Diseases Control and prevention, 68(LL - 2).

32. Battacolo JL (2018) Causes and Risk factors of Gonorrhea infection. Asian Journal of Urology 3(4): 56-60.

33. Fan W, Zhang Q (2012) Risk factors for male patients with gonorrhoea complicated by inflammation of the par urethral glands around the external urethral orifice. Int J STD AIDS 23(6): 400-402.

34. Monif GR, Baker DA (2010) Infectious Diseases in Obstetrics and Gynecology, Obstetric medicine, $5^{\text {th }}$ (Edn.), New York, NY 3: 126.

35. Mastromarino P, Hemalatha R, Bartonetti A, Cinque B, Cifone MG, et al. (2014) Biological control of vaginosis to improve reproductive health. Indian J Med Res 140 (S1): S91-S97.

36. Paavonen J (1996) Immunopathogenesis of PID and infertility - What we know and what shall we do? J Br Fer Soc 1(1): 42-45.

37. Kaur K, Prabha V (2014) Sperm agglutinating Escherichia coli and its role in infertility: In vivo study. Microb pathog 69-70: 33-38.

38. Sachdev D, Lal Pated A, Sonkar SC, Kumari I, Saluya D (2015) Diagnosis of Neisseria gonorrhoeae using molecular Beacon. Biomed Res Int.

39. Cohen MS, Cannon JG (1999) Human experimentation with Neisseria gonorrhoeae: progress and goals. J Infect Dis 179(S2): S375-S379.

40. Burnham RC, Plummer F, Slaney L, Rand F, DeWitt W (1985) Correlation of auxotype and protein I type with an expression of disease due to Neisseria gonorrhoeae. J Infect Dis 152(2): 339-343.

41. Gilberto J Ramos (2012) The role of Chlamydia trachomatis in male infertility. Intech Open 245-257.

42. WHO (2010) WHO Laboratory manual for the examination of Human Semen and sperm-cervical mucus interaction, $5^{\text {th }}($ Edn.), Cambridge University Press, pp: 189- 200.

43. Wong B (2018) Gonorrhoea workup. Medscape 6(2): 11

44. CDC (2015) Sexually Transmitted Diseases Treatment Guidelines. Centres for Disease Control and Prevention MMWR 64(RR 3): 1-137.

45. Paul G Engelkirk, Janet Duben-Engelkirk (2008) Laboratory Diagnosis of Infectious Disease. Essential Microbiology-Lippincott William and Wilkins, $5^{\text {th }}$ (Edn.), A review, pp: 234-237.

46. Llmar (2007) Sexually Transmitted Bacterial pathogen for which there is increasing Antimicrobial Susceptibility testing. Journal Manual for identification and Antimicrobial Susceptibility testing. Centers for Disease Control and Prevention. 


\section{Open Access Journal of Microbiology \& Biotechnology}

47. Brill RJ (2010) Sexually Transmitted Infection in men. Prim Care 37(3): 509-525.

48. CDC (2017) American Social Health Association. Center for Disease Control and Prevention, pp: 783-987

49. Leistikow H, Holmes KK, Mardh PA, Sparling PF, Lemon
SM (1882) Biology of Neisseria gonorrhoeae: Sexually transmitted diseases, $3^{\text {rd }}$ (Edn.), McGraw Hill, New York, NY, pp: 433-449.

50. Zaidi AA, Aral SO, Reynolds GH, Blount JH, Jones OG, et al. (1983) Gonorrhea in the United States: 1967-1979. Sex Transm Dis 10(2): 72-76. 